\title{
Using Blockchain-Enabled Solutions as SDG Accelerators in the International Development Space
}

\author{
Ahmet Faruk Aysan ${ }^{1, *(D)}$, Fouad Bergigui ${ }^{2}(\mathbb{D})$ and Mustafa Disli $^{1}$ (D) \\ 1 College of Islamic Studies, Hamad Bin Khalifa University, Doha 34110, Qatar; mdisli@hbku.edu.qa \\ 2 SDI Global Consult, Dover, DE 19901, USA; f.bergigui@sdi.world \\ * Correspondence: aaysan@hbku.edu.qa
}

Citation: Aysan, A.F.; Bergigui, F.; Disli, M. Using Blockchain-Enabled Solutions as SDG Accelerators in the International Development Space. Sustainability 2021, 13, 4025 .

https://doi.org/10.3390/su13074025

Academic Editor: Shervin Hashemi

Received: 2 March 2021

Accepted: 29 March 2021

Published: 5 April 2021

Publisher's Note: MDPI stays neutral with regard to jurisdictional claims in published maps and institutional affiliations.

Copyright: (c) 2021 by the authors. Licensee MDPI, Basel, Switzerland. This article is an open access article distributed under the terms and conditions of the Creative Commons Attribution (CC BY) license (https:// creativecommons.org/licenses/by/ $4.0 /)$.

\begin{abstract}
As the world is striving to recover from the shockwaves triggered by the COVID-19 crisis, all hands are needed on deck to transition towards green recovery and make peace with nature as prerequisites of a global sustainable development pathway. In this paper, we examine the blockchain hype, the gaps in the knowledge, and the tools needed to build promising use cases for blockchain technology to accelerate global efforts in this decade of action towards achieving the SDGs. We attempt to break the "hype cycle" portraying blockchain's superiority by navigating a rational blockchain use case development approach. By prototyping an SDG Acceleration Scorecard to use blockchain-enabled solutions as SDG accelerators, we aim to provide useful insights towards developing an integrated approach that is fit-for-purpose to guide organizations and practitioners in their quest to make informed decisions to design and implement blockchain-backed solutions as SDG accelerators. Acknowledging the limitations in prototyping such tools, we believe these are minimally viable products and should be considered as living tools that can further evolve as the blockchain technology matures, its pace of adoption increases, lessons are learned, and good practices and standards are widely shared and internalized by teams and organizations working on innovation for development.
\end{abstract}

Keywords: blockchain; SDGs; innovation; COVID-19; green recovery; scorecard

JEL Classification: O16; O19; O35; P16; Q01; Q56

\section{Introduction}

As we enter the decade of action to achieve the SDGs by 2030, the international community is facing unprecedented challenges to accelerate the pace towards meeting national sustainable development targets, at a time where the very development gains won over the last decades continue to be reversed by the aftershocks of the COVID-19 crisis [1]. If new technologies are going to contribute to the necessary transformation, there have to be adequate tools, methodologies, and standards to navigate the blockchain "hype cycle" and to move from a generalized "let's blockchain it" approach towards a rational narrative that is evidence-based.

Despite blockchain's promising potential, the reality can be challenging, there are not enough data, blockchain-backed applications for social impact are under-studied, and claims that blockchain-backed solutions can yield superior results when compared to other alternatives are yet to be supported by evidence [2]. While we can agree that blockchain has the potential to trigger disruptive innovations, we can also agree that the technology is not yet mature and that there is still a gap in terms of approaches and tools needed to develop blockchain use cases, evaluate blockchain applications, monitor experiments, mitigate associated risks, and manage organizational changes to galvanize innovation-readiness within organizations considering adopting blockchain technology and running use case experiments [3]. It is only by filling the existing gaps that we can make a stronger case for using blockchain as an SDG accelerator. 
We can argue that the comparative advantages of blockchain can be explained by its ability to address the issue of trust within the global financial system in the aftermath of the 2008 global financial crisis [4]. Looking into the current practice among the key players in both public and permissioned blockchains, there is a costly race among early adopters towards becoming the standard and prototyping blockchain applications to do things other technologies cannot. Against this background, we can look at blockchain as a package of technologies and approaches that can be used to open up new opportunities for users to manage transactions, exchange values, and maintain digital trust [5].

In one forecast, the business value of blockchain was predicted to climb up to $\$ 176$ billion by 2025, before skyrocketing to \$3.1 trillion by 2030 [5]. Nevertheless, blockchain is yet to match the hype, despite its potential. The maturity of blockchain technology is expected to facilitate its wider adoption, widen the scope for its utilization, and consequently foster blockchain's ability to stimulate successful disruptions. This is in line with the need to stimulate business processes, enable regulatory frameworks, and trigger the necessary cultural shift and the underlying organizational structures.

In this paper, we examine how various blockchain solutions should be ideated, designed, and applied, and how related implementation choices can be made in an attempt to define a structured approach to develop effective use cases for blockchain applications in the fields of sustainable development and green recovery. We then identify potential gaps in terms of monitoring and evaluation, risks, and ethical considerations in order to propose tools to assess the impacts of blockchain-powered applications and manage the associated potential risks and ethical considerations. We also explore the extent to which the use of blockchain influences business processes and identify structured ways to manage transformational change within organizations. Individuals and organizations designing and experimenting blockchain-based solutions for sustainable development may benefit from the tools we have prototyped in this study. In this regard, we propose the SDG Acceleration Scorecard and the integrated approach to using blockchain-enabled solutions as SDG accelerators.

In doing so, we aim to stimulate a discourse around effectively harnessing the disruptive potential of blockchain as an SDG accelerator. This work will support development workers and organizations experimenting or willing to experiment with blockchain-enabled applications to address various challenges related to the achievement of the sustainable development goals by providing a practical toolbox. Such a toolbox will (i) facilitate the deployment of structured approaches and meaningful tools to build promising use cases for blockchain applications; (ii) monitor and evaluate the results of blockchain-enabled development interventions; (iii) assess and mitigate the risks associated with blockchain technology; (iv) manage organizational change and nurture a culture of change that will promote early and rational adoption of disruptive innovations.

\section{Blockchain and Token Solutions}

\subsection{Blockchain Technology}

Blockchain is an open-source technology that excludes the traditional third parties by relying on collective verification, thus offering a great alternative in terms of costs, traceability, security, and speed. When two financial entities such as banks receive a request to transfer money from one account to another, they have to update the balances of their respective customers. This costly and time-consuming coordination and synchronization exercise can be simplified on a blockchain by using a single ledger of transactions reflecting a single version of records instead of two different databases [6]. Blockchain applications go beyond finance and are growing to encompass a myriad of use cases $[7,8]$.

Blockchains can be designed either as private or public; while decentralization remains a common denominator to both forms, there is a key difference in the level of access granted to participants [9]. In the case of a public blockchain, participants are typically encouraged to join the network through an incentivizing mechanism, such as in the case of Bitcoin [10]; anyone can join the network and decentralization is pushed to the fullest extent [9]. On the 
other hand, private or permissioned blockchains are closed networks where participants face restrictions in terms of who can write data and who can read it. Hence, while public blockchains maximize the anonymity, permissioned blockchains know the identities of their participants and determine which information they should or should not have access to [11].

While perceptions suggest that public and permissioned blockchains are competing with each other, they have different offerings and could be rather complementary in terms of the solutions they offer [11]. Public blockchains offer high security, an open environment, anonymity, and no restrictions, whereas private blockchains prioritize privacy, high efficiency, and stability. We can argue that permissionless blockchains empower the user by pushing transparency and decentralization to their full extents, while permissioned blockchains empower enterprises instead of individual employees [11,12]. The convergence of public and private blockchains is expected to pave the way for virtual ecosystems where a wide range of players can collaborate in a secure and auditable way [7].

The question remains of which blockchain is better for which applications? Indeed, public and private blockchains have distinct use cases. In general terms, public blockchains address business-to-consumer scenarios, while private blockchains are more applicable to business-to-business relationships, with some shared infrastructure between businesses [12]. The transparency and security features of public blockchains make them more suitable for developing blockchain-enabled solutions serving larger communities where trust is a key concern [9]. They are a viable option in situations where all users should be treated equally and when the protection of users' anonymity brings added value to the solution [12]. There are, however, some concerns about whether confidential data should be recorded on a public blockchain, assuming that the encryption could be hacked one day [9].

In the world of private blockchains, there are quite opposing concerns, since the players are reluctant to publicly share their business data. This is more appealing to financial institutions and corporations so they can know and predetermine who has access to what [9]. The downside though is that trust comes down to the credibility of the authorized nodes, as well as a relatively higher vulnerability to malicious attacks [9]. As blockchain technology keeps evolving, hybrid solutions could perhaps offer the best of both options by bringing together trust and security alongside efficiency and speed [9]. Given the current momentum in adopting blockchain applications across a large spectrum of industries, blockchain technology can only increase in popularity as the world enters the uncharted territories of the "new normal" in the post-COVID-19 era, where technologies are poised to play an extremely important role in redefining "business as usual".

\subsection{Tokenization}

Blockchain technology offers a myriad of value through a frictionless process of immutable and transparent records and through converting assets into digital tokens (i.e., tokenization) with smart contracts. These features offer solutions that are particularly suited to addressing challenges in the implementation of SDGs. Special importance is given to "impact tokens", which represent a group of tokens designed to unlock investments for projects with positive social and environmental impacts [13]. The deployment of these impact tokens in blockchain offers new mechanisms to improve ESG ratings, as it offers proof that a particular investment has delivered a positive impact [14]. A key advantage of tokenization is that it offers traceability across the supply chain ecosystem. In this regard, token-based incentive schemes should further broaden the accessibility of blockchainbased solutions for sustainable development, in alignment with UN SDGs. Furthermore, as suggested by Uzsoki and Guerdat [13], the UN or other international bodies could facilitate the adoption of impact tokens by setting common standards that set out both their characteristics and achievement of SDGs.

Supported by UN World Food Program, Fishcoin is a blockchain-based data-sharing platform that incentivizes catch registration and data-sharing across the seafood supply 
chain [15]. Data contribution to the blockchain platform is rewarded by tokens, which can be exchanged for mobile phone credit. Amply, a pilot project in South Africa that has been funded by UNICEF Innovation Fund and Innovation Edge, tracks school attendance by providing children with self-sovereign digital identities on the blockchain. When a teacher confirms attendance on a mobile application, a token is generated that the school can redeem for further subsidies. Moeda is a blockchain-based cooperative banking system that leverages on a fiat-pegged digital token. The Moeda initiative facilitates access to finance to unbanked and underbanked entrepreneurs, whereas impact investors are able to keep track of their investments.

\section{Methodology}

To prototype an integrated approach for this research, we will go through several stages. First, we will define the minimum viable product for the integrated approach by examining the available literature using four thematic clusters: building blockchain use cases, monitoring and evaluation of blockchain-based applications, risks and ethical considerations associated with blockchain-powered experiments, and change management in organizations deploying blockchain-based solutions. Second, the integrated approach built on the initial findings from the literature review will be further improved based on our observations and the inputs shared by resource persons approached to conduct this research.

During the last stage, we will finally propose an integrated approach together with the SDG Acceleration Scorecard to assess the potential for SDG acceleration. While we recognize the limitations of this work and the need to conduct further field investigations to explore the proof of concept by reaching out to a larger number of respondents to gather and analyze critical data, we believe that the proposed package composed of the integrated approach and the SDG Acceleration Scorecard is a necessary first step. Such tools should be considered as "living tools" that can be further improved as blockchain technology matures, based on the feedback from end-users as they domesticate and internalize these tools to develop meaningful blockchain solutions.

We consider four thematic clusters according to which we will test the following research questions and assumptions in an attempt to verify their validity (Table 1).

\subsection{Building a Use Case}

The low experimentation costs of blockchain solutions driven by platforms made available via service providers known as blockchain as a service (BaaS) offerings [16], combined with the increasingly complex nature of delivering development and humanitarian interventions in a time where both developing and developed countries are pursuing their sustainable development targets, has stimulated many investments in use cases for blockchain solutions in a wide array of contexts. Here, we are looking beyond the hype at the fundamental question of what can be and what cannot be solved by blockchain? We can also agree that given the scarcity of finances available for development, the increasing demand and challenges faced by organizations and practitioners in today's world to deliver effective yet lasting results, the return on investment of innovation experiments, including blockchain, should not be limited to the proof of concept but should also consider impacts at scale.

While blockchain can be the appropriate choice in some cases, other conventional technologies might be more appropriate in other situations [17]. Given the blockchain hype, we consider that the critical starting point to make a use case for a given blockchain application is to avoid at all costs developing a blockchain solution when there is no problem to be solved with it. The specific problems that need to be fixed and the feasibility of the blockchain solution should be rigorously examined while building a promising use case for blockchain [18]. Other criteria to consider could include the realities on the ground in line with the specific development or humanitarian contexts for which a blockchain 
solution is sought, the capabilities of the staff involved in the blockchain experiment, the flexibility of the applicable business processes, and the underlying managerial structures.

Table 1. The thematic clusters proposed to prototype the integrated approach.

\begin{tabular}{|c|c|}
\hline Thematic Cluster & Research Questions and Assumptions \\
\hline Building a use case & $\begin{array}{l}\text { What can be solved and what cannot be solved by blockchain? Which structured approach } \\
\text { should be followed to understand the potential of blockchain in a given context and design } \\
\text { specific and promising use cases? We assume that the solution should not come before the } \\
\text { problem. To make a meaningful use case for blockchain there are many boxes to tick. }\end{array}$ \\
\hline Risks and ethical consideration & $\begin{array}{l}\text { Are blockchain applications in sustainable development risk-free? What are the potential } \\
\text { risks associated with the utilization of this technology and which ethical reflections should } \\
\text { be taken into consideration? We assume that there is a need to explore ethical considerations } \\
\text { around the use of blockchain to determine potential risks for development professionals to } \\
\text { make informed decisions and come up with appropriate mitigation measures. }\end{array}$ \\
\hline Monitoring and Evaluation & $\begin{array}{l}\text { Which structured approach and tools can be used to monitor and evaluate blockchain } \\
\text { applications? Looking beyond pilots, can blockchain solutions deliver impacts at scale? } \\
\text { How can we measure such impacts? We assume that while blockchain applications are } \\
\text { contributing to solve some of the challenges around the implementation of the SDGs, this } \\
\text { has been mostly demonstrated and reported in the available literature through pilot } \\
\text { applications that were not replicated at a larger scale. To better assess blockchain's impact } \\
\text { on the SDGs, to examine whether or not a given result can be fully attributed to the } \\
\text { blockchain, or if blockchain rather partially contributed to such a result, we assume that } \\
\text { there is a need to develop adequate tools to support monitoring and evaluation of different } \\
\text { blockchain experiments. Such tools should not only track progress but should also provide } \\
\text { a standardized platform to ensure comparability and assess criteria of relevance, } \\
\text { effectiveness, efficiency, sustainability, and impact, in addition to gender equality. }\end{array}$ \\
\hline Change management & $\begin{array}{l}\text { What about change management in organizations adopting blockchain? How does } \\
\text { embracing innovation, in the case of blockchain applications, affect the way development } \\
\text { and humanitarian organizations do business? We assume that change management is a } \\
\text { dynamic process that needs to be understood to enable organizations to develop an } \\
\text { evolutionary adaptation approach to navigate the transition, address resistance to change, } \\
\text { and nurture innovation. }\end{array}$ \\
\hline
\end{tabular}

Another vital factor is the coordination among stakeholders involved in the design and implementation of a given blockchain solution. To design viable blockchain solutions, due consideration should also be given to the ability to manage technology constraints through trade-offs, to convince various stakeholders of the relevance of the blockchain use case, to cooperate with other players, and to comply with the applicable regulations and standards on a case-by-case basis.

For this research, we have adapted the stages defined in the blockchain use case development method (BUD) proposed by Fridgen et al. [19] using a design thinking process [20]. We argue that these stages will evolve to reflect the latest developments in blockchain as the technology matures and reaches a stage of producing impacts at scale.

Step 1-Understand blockchain

We assume that the focus here should not be on understating the technology itself, but on what can be done with it. This also takes into consideration the capabilities organizations should have to design and implement blockchain solutions.

Step 2-Define the problem you are trying to solve with blockchain

Here we need to clearly define the challenge that we are trying to overcome with blockchain. Individuals and organizations need to consider feasibility aspects, while various organizational implications with regard to business processes and change management should also be taken into consideration. Guiding questions can be provided, such as:

- What is the exact problem you are trying to solve?

- How does it affect your sustainable development context? 
- Which capabilities do you need to develop a blockchain solution to address this problem? Step 3-Pitch your ideas

We assume that while teams can consider existing blockchain use cases in their fields of interest, they should only use such cases for inspiration and come up with their innovative ideas by brainstorming and working together in cross-functional teams. Guiding questions can be provided, such as [21]:

- Are transparency and traceability important in your context?

- Do you need a centralized or decentralized solution?

- Which transactions require trusted partners or processes?

- Which intermediaries can be eliminated?

- Will you reduce transaction time for end-to-end processes with blockchain?

- Which costs can be reduced?

- Which data are most sensitive and which data will be stored?

- How much control do you need for stored data?

- Who are your potential end-users?

- What transformational change can result from using blockchain in your organization? Step 4-Build Consensus

We assume that while consensus protocols make blockchain platforms immutable, achieving such consensus may require huge computational power and large amounts of energy, while design choices can have implications on how much time is needed for consensus and the degree of decentralization.

- $\quad$ To what extent are timing and decentralization critical factors in your context?

- How much computational power is needed to achieve consensus? Can it be reduced?

- Which consensus protocols can be used?

Step 5-Prototype the solution

We assume that issues of efficiency, scalability, performance, and impact should be factored-in while defining the parameters for any given blockchain prototype. Guiding questions may include:

- What is the cost of developing this blockchain solution?

- What would be the return on investment?

- Which blockchain platform should be used in your case (public, private, or hybrid)?

Step 6-Test and iterate

We assume that blockchain solutions should be tested with potential end-users to identify residual issues that will be taken back to the prototyping phase through an iterative process.

Figure 1 summarizes the steps in the blockchain use case development method.

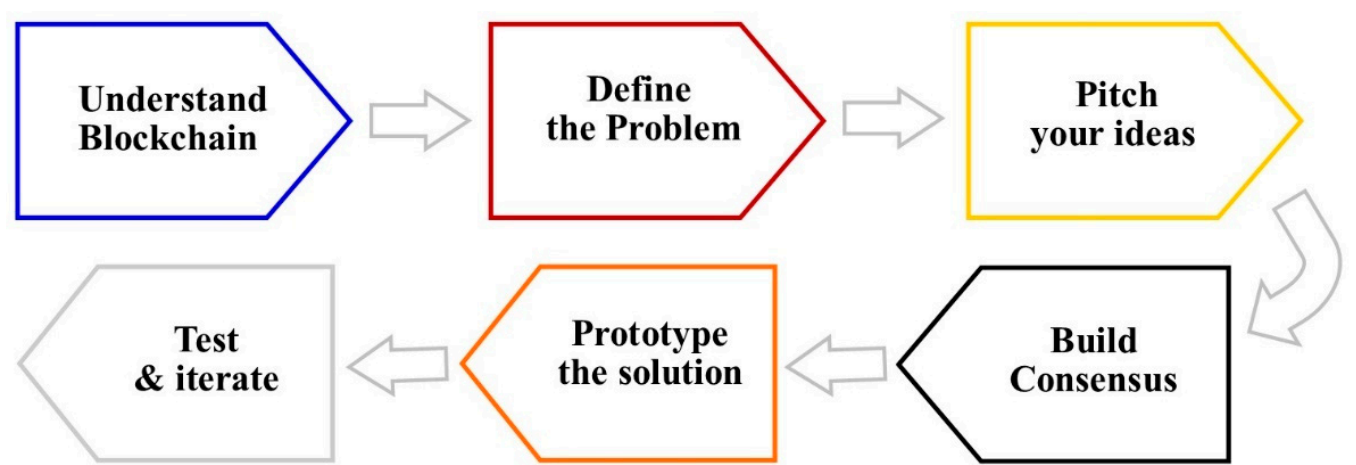

Figure 1. Presentation of the blockchain use case development method. Source: The author based on Fridgen et al. [19]. 


\subsection{Risks and Ethical Considerations}

We can praise blockchain for its disruptive potential and all the benefits it can bring into a given use case. Nevertheless, we can also agree that there is no return on investment without risks. Organizations and practitioners must avoid at all costs creating more problems than the ones a blockchain application is designed to solve. Given the complex nature of blockchain technology, it is not an easy task to assess the underlying risks, especially in the absence of standards and regulations [22]. For this research, we adapted the frameworks for risk analysis and management [23-25]:

- Analyze (identify core risks and assess their potential impact and likelihood);

- Manage (develop proper management responses to mitigate risks);

- Anticipate (detect new risks that are arising from various ethical considerations).

\subsubsection{Risk Analysis}

At the design stage, there are no blockchain standards, as the technology is not mature yet. Lessons learned and good practices should be coded into standards to minimize risks and pave the way towards enhanced cooperation. We assume that the interoperability between blockchain platforms will remain a key issue, and thus represents a key risk. Other technical risks may potentially stem from design-related choices, for instance whether forking another version of the ledger is allowed or whether automated actions are built-in on a blockchain infrastructure.

Implementation comes with its own set of risks. Consensus-building algorithms can cause serious delays given the nature of participatory processes. High maintenance costs can cause projects to fail and scalability might not be achieved through economies of scale. Cybersecurity risks and fraud are key challenges for shared technological infrastructure that can originate from the outside or from within. While the likelihood of outside attacks appears to be minimal, risks stem from the irreversible nature of internally produced mistakes, such as sharing an erroneous code across the network or building consensus on a false entry. Updates and maintenance managed by third parties may qualify as potential risks as well, especially if the skills required and the availability of service providers are in low supply.

Other risks in general terms are linked to the constantly evolving blockchain regulations in various jurisdictions, the cost of technology updates, competition from other disruptive innovations, and the ecological footprint of blockchain given its energy consumption. In general terms, the more we understand blockchain and the more we understand the problem we are trying to solve with blockchain, the higher our chance of coming up with the most appropriate risk mitigation measures.

\subsubsection{Risk Management}

We consider three risk categories that can be analyzed at different stages before, during, and after the implementation of the blockchain-backed solution.

\section{Preventable Risks}

Here, we are looking at internal and avoidable risks arising from within organizations and teams. With blockchain, the need for human interventions can be reduced when using automated processes and smart contracts, which in return can decrease the likelihood of alteration of records or corruption of people involved. However, risks may stem from the irreversible nature of internally produced mistakes, such as sharing an erroneous code across a network or building consensus on a false entry. We assume that active prevention through a rules-based control model is the most suitable risk management response to risks listed under this category, which can be achieved by monitoring business processes and by guiding human-made decisions related to blockchain applications. 


\section{Strategic Risks}

These are risks organizations and teams are willing to take to achieve high targets. Here, the managerial response is rather towards reducing the likelihood for risks to occur and containing such risks in the case they materialize. While blockchain can minimize risks related to compliance and litigation, early investors, driven by an appetite for innovation and to be pioneers their fields of practice, may see their projects seriously delayed by consensus-building algorithms given the nature of the participatory processes, or their projects may even fail due to poor buy-in from their stakeholders and high maintenance and scalability costs.

\section{External Risks}

These are consequences of events happening in the outside world that cannot be accurately forecasted, and over which organizations and individuals have no control whatsoever. Proper mechanisms should be set in place to track such risks and mitigate their impacts to the extent possible using appropriate managerial responses. Although the likelihood of outside attacks appears to be minimal, cybersecurity risks are a key challenge for shared technological infrastructure. Updates and maintenance managed by third parties may qualify as potential risks as well, especially if the skills required and the availability of service providers are in low supply. Other risks in general terms are linked to the legal-technology mismatch due to the constantly evolving blockchain regulations in various jurisdictions, in addition to the cost of technology updates and competition from other disruptive innovations.

\subsubsection{Ethical Considerations}

We can argue that ethics made a late appearance in the blockchain sphere because it is seemingly hard to understand how the technology works. Several ethical considerations need to be addressed. Due to massive energy consumption, public blockchains based on mining can potentially have a significant impact on the environment. The annual total electricity consumption of bitcoin assuming constant power usage over one year is estimated at 77.38 TWH [26]. This number should be understood as an educated estimate, as it is not possible to accurately calculate the electricity consumption of bitcoin processes.

The hyper-efficiency that is indeed a valuable comparative advantage of blockchain applications can disrupt the jobs of many people involved, for example those working in data maintenance [27]. Additionally, blockchain can over-promise and fail to meet expectations or inadvertently facilitate crime-related transactions and oppressive conduct [28]. Blockchain can be used for money laundering in illicit business activities, such as weapons and drugs or other related transactions [29].

Equally important is the way we design blockchain applications based on tradeoffs, which can have far-reaching consequences [30]. Such trade-offs can codify biases and exacerbate social dynamics within a given community, while the transparent nature of blockchain information can put persons at risk because of their ethnicity, religious background, or sexual orientation [30]. The same applies to the immutability of digital identity records if a person is threatened and requires anonymity or a new identity, or if erroneous data are recorded. If there is no way of retrieving a lost private key, this might simply mean that a person loses control over their digitally recorded assets. When the encryption algorithms used are outdated, sensitive information might be exposed and transactions can be forged [30].

Due consideration should also be given to data privacy. In the absence of natural or legal persons to fulfill data privacy requirements as data controllers, given the decentralized nature of blockchain platforms, one of the key challenges for blockchain-powered solutions is how to define controllership over data. Another key challenge is the underlying assumption that data can be erased should such a need arise based on data privacy requirements [31]. 
There is a steep compliance curve ahead for blockchain practitioners to comply with the requirements stipulated in General Data Protection Regulations (GDPR). In order to build privacy-compliant use cases of blockchain-backed solutions using GDPR lenses, silos should be broken down through proactive dialogue involving technology disruptors, developers, and regulators to address key challenges related to defining data controllers or deleting personal data [32]. By doing so, more privacy-friendly operational procedures can be codified into the process of building blockchain use cases.

\subsection{Monitoring and Evaluation (MEE)}

\subsubsection{Monitoring}

We argue that the core ingredients to successfully monitor blockchain interventions would be for organizations to have clear monitoring policies in place, for individuals to have clearly defined roles and responsibilities in the monitoring process, and to have a first-hand understanding of the operational context in which a given blockchain-powered application is being deployed. Appropriate M\&E frameworks should be developed to track specific project outputs, especially related to the annual targets that blockchain projects expect to achieve in line with their result matrices. Monitoring actions should capture and analyze data to report on the progress towards outcomes, impeding factors, partners' roles in achieving the expected results, and lessons learned to develop and disseminate knowledge products. While blockchain experiments for social good remain largely understudied, such knowledge products are undoubtedly needed by the global community given the scarcity of data available to identify good practices. Additionally, monitoring data and reports are key resources that can be used to inform decision-making and future experiments.

For this research, we propose a three-step approach for monitoring blockchain projects, namely data gathering, analysis, and reporting:

- Collecting data through available tools. Data can be collected based on field investigations and through different reporting mechanisms, then triangulated for validation and discussed via participatory processes, such as during coordination meetings with partners;

- Analyzing the data gathered to extract useful information, identify patterns, and detect bottlenecks. Data interpretation in terms of what needs to be done may be used to generate useful and user-friendly insights to guide decision-makers;

- Reporting to inform future decisions. Stakeholders and partners involved in blockchain projects can be guided to make informed decisions to ensure the project is on track to deliver their expected results.

\subsubsection{Evaluation}

Since their adoption in 1991 [33], the five OECD DAC criteria are the most referenced and used standards for evaluating international development interventions. For many years, evaluation professionals have considered relevance, effectiveness, efficiency, impact, and sustainability as the standards to assess development interventions. The main issue here seems to be how these criteria should be used, as one mainstream mistake is to start using such criteria in a mostly mechanical way by "ticking boxes" instead of using them to support a non-linear critical analysis [34]. There are ongoing discussions regarding the extent to which the DAC criteria should be revised to capture the new paradigms of sustainable development. We can argue that not every evaluation should cover all 5 requirements; even if this is the case, not all criteria should be analyzed in the same depth, and most importantly these criteria may not be entirely suitable for the needs of today [34]. We are looking here into disruptive innovations and specifically blockchainenabled applications for sustainable development to extract key guiding criteria, which evaluation professionals could consider when evaluating blockchain-backed projects.

One common critique of blockchain applications is the lack of evidence to support claims for disruptive results. This can be partially explained by the fact that the technol- 
ogy is not mature yet, or that lessons learned from blockchain experiments are poorly documented. As a result, we can argue that the hype has been driven to some extent by a "fear of missing out" bubble based on proof-of-concept experiments design to make headlines [35]. With that being said, there is a promising potential for blockchain use cases to have a transformative business impact by improving productivity and quality, increasing transparency, and reinventing products and processes [36], hence the need to fill in the gaps by developing proper M\&E evaluation frameworks for blockchain applications for international development.

After developing a promising use case for blockchain, securing the support of various partners, unlocking the necessary funding, and before kicking-off the implementation of a given blockchain-powered solution, there is a need to set up robust M\&E frameworks. Organizations might consider hiring full-time M\&E practitioners or investing in diffuse M\&E skills throughout their workforce assigned to design and implement blockchain-based applications. However, various considerations make it difficult to perform M\&E functions for sustainable development interventions driven by innovative processes. For instance, the uncertainty associated with innovation-based solutions, the speculative nature of the results one should expect down the road, and the hard-to-measure nature of innovation-driven benefits, such as stimulating an appetite for a diffuse "business as unusual" culture, are all vital aspects to consider while monitoring and evaluating blockchain-centered experiments.

Before implementation, certain criteria need to be considered for blockchain practitioners to come up with the right decisions [37]. In terms of governance, for instance, it is vital to identify who controls access to the blockchain application, who makes decisions, and who enforces such decisions to alter the blockchain solution as the needs evolve in a given development or humanitarian context. One of the main aims for developers of blockchain applications in sustainable development contexts is to minimize their power consumption and ecological footprint [26]. In this regard, choosing the right consensus scheme and offering the right incentives can enhance the acceptability, and thus the success, of blockchain-enabled solutions. To decide on the right type of blockchain platform to deploy, organizations need to take into consideration whether the data are meant to be accessible publicly, in a restricted circle, or as a combination of both.

For this research, we have adapted the evaluation framework that was developed by Fridgen et al. [38] to evaluate the applicability of blockchain technology in the public sector. We argue that this framework is not only suitable for the evaluation of blockchain use cases, but also for the provision of a set of evaluation criteria to consider while conducting mid-term or final evaluations of blockchain projects for sustainable development. Here, we consider 3 sets of criteria, namely technical, functional, and legal criteria.

\section{Technical Criteria}

In this set, we have included 4 parameters, namely performance, scalability, security, and usability.

\section{Performance}

Due consideration should be given to how fast transactions can be operated within a blockchain network. Furthermore, duration and latency need to be verified using available metrics, such as the number of blocks and the size of transactions.

\section{Scalability}

This is a key element given that blockchain experiments are usually designed to be tested in small pilot cases. Once the candidate blockchain applications deliver proof of the concept and graduate from the experimentation phase, they are expected to be applicable on a larger scale to address international development. It is, thus, critical to factor-in scalability parameters such as the network size, number of possible nodes, and transactions needed down the road on a larger scale.

Blockchain-powered solutions leverage the network effects of the investment universe by unlocking funds from small investors. Network effects through blockchain technology 
are achieved without the disruptive consequences the presence of an intermediary brings with it. In this regard, Catalini and Gans [39] point out that decentralized networks reduce transaction costs and enable network effects without being subject to monopoly pricing and control. Blockchain has the power to enable an accelerated realization of SDGs, as it helps to connect and integrate different databases and information flows [40]. The decentralized principle of value creation through blockchain-based architectures will benefit sustainable development by facilitating simultaneous collaboration and competition among partners within the loop [41]. The network effects result in a positive feedback loop, whereby the services offered become strengthened with the adoption of the network [42]. It is evident that blockchain network effects will manifest at an increasing pace when the ecosystem becomes more valuable to its participants as more people partake in it.

\section{Security}

We can assume that a fair number of development solutions have been designed as blockchain applications given the enhanced security features of blockchain technology. This points out the need to carefully consider the security features provided by a given blockchain application and assess whether it answers specific contextual requirements related to the security of data and transactions.

\section{Usability}

User-friendly applications tend to be more accepted. The ease of use is a critical parameter to consider, which comes down to ensuring that everyone can benefit from the blockchain solution and that no one is left behind. Important details to factor-in can range from internet traffic to the ability to fork new features, the available tools and languages, and the number of possible users.

\section{Functional Criteria}

This set includes criteria related to the R\&D costs required to build a blockchainbacked solution, implement it, and ensure its operationalization and maintenance, in addition to other criteria related to the flexibility and transparency of the process.

\section{Costs}

Some blockchain applications may require heavy transactions and storage of data, implying high costs. This should be duly considered while budgeting to include not only the prototyping and deployment of the blockchain application, but also its operational and maintenance costs.

\section{Flexibility}

Another important feature in international development setups is the ability of blockchain-powered pilots to be replicated or altered in a way to serve new needs or address new challenges. For instance, $\mathrm{ff}$ a blockchain platform is only meant to be deployed in a restricted area, such limitations are to be considered before any investments are made upfront.

\section{Transparency}

It is important to find the right trade-off between transparency and other outputs. Confidentially can be increased by storing encrypted data, however it will reduce transparency and performance. Transparency can be in conflict with commercial confidentiality, such as in cases where competitors exist [17].

\section{Legal Criteria}

This set includes criteria related to data privacy, legal procurement frameworks, employee protection rights, and other legal regulations. Here, we highlight various legal considerations applicable in the jurisdictions and to the entities involved in blockchain experiments. These include requirements for data privacy, contractual modalities specified 
in procurement laws, employment protection rights, and any other legal instruments that are potentially applicable to blockchain within a specific jurisdiction.

\subsection{Change Management}

We can argue that blockchain technology stimulates a progressive evolution in the ways business is conducted. Development organizations and practitioners running various innovation experiments and aspiring to harness the disruptive potential of blockchain are facing the challenge of managing the change towards a "new normal". New avenues and meaningful methods are being explored to nurture a culture of innovation, keep risks at acceptable levels, and address pockets of resistance regarding both organizations and individuals being able to follow the trajectory of evolutionary adaptation and achieve transformational change in terms of doing business [5]. Blockchain should not be considered as a technology option but should be understood in terms of its ability to address specific issues and deliver precise solutions. Organizations and individuals should also consider experiments where they can explore decentralized business processes and allocate staff who have both technological and technical capabilities [5].

We also argue that organizations and individuals embracing the disruption wave brought about by blockchain technology need to be change-adept. When an organization invests in a blockchain application, it has already identified a problem and came up with a use case for blockchain as a solution. In this respect, managers will need to set up innovation-prone environments to ensure effectiveness and efficiency, reinforce collaboration, address siloed-behaviors, provide leadership and coaching, and avail resources needed for the blockchain-enabled solution to deliver on its promises. Here, the focus is on the capability of the organizations to implement a blockchain-backed solution. In fact, without a structured approach to implementation, an innovative solution is likely to fail. We assume that change management is a dynamic process that needs to be understood in order to enable organizations to develop an evolutionary adaptation approach to navigate the transition, address resistance to change, and nurture innovation.

For this research, we will focus on change at the individual level as the cornerstone for change to happen at the organizational level. The following change management approach was inspired by the ADKAR (awareness, desire, knowledge, ability and reinforcement) and Kurt Lewin change management models [43,44]:

\section{Unfreeze}

At this initial stage, the focus should be on raising awareness beyond the hype to visualize potential opportunities to adopt blockchain-based solutions and seize the most promising ones.

\section{Change}

This is an intermediary stage where the spotlight is on motivating individuals and teams to design blockchain-powered solutions. This includes coaching, managing resistance, fostering readiness to become a change agent, as well as investing in core capabilities through learning and practice for individuals and teams to acquire a wide spectrum of skills deemed necessary to implement blockchain projects.

\section{Refreeze the change}

This is the final stage, where the focus in on re-calibration through corrective actions whenever applicable, but also on measuring achievements and celebrating success.

\section{Results}

\subsection{SDG Acceleration Scorecard}

Given the current hype, one can argue that in general terms, development practitioners and organizations tend to act prematurely to address a giving problem without necessarily being aware of the alternatives and without running proper assessments that would or would not prioritize blockchain as the best way forward. Even after an assessment 
indicates that a blockchain solution is the best option, one can still do more to harness not only the direct benefits to address the initial problem for which a blockchain solution is needed, but equally important are the wider sustainable development co-benefits to which such blockchain solutions can contribute. Given the interlinked nature of the SDGs, one blockchain-backed solution can contribute to more than just one SDG at a time. In other terms, one should think beyond the simple issue to be solved by a blockchain application. While such an application can solve a specific problem, it can also contribute to achieving other development targets if properly designed and implemented, hence the SDG acceleration effect. That is why we truly believe that for blockchain-powered applications to deliver wider development co-benefits, it is critical to assess their SDG acceleration potential by taking into consideration an initial set of criteria and indicators related to use case development, monitoring and evaluation, risks and ethical considerations, and change management. We refer to Table 2, which showcases examples through which dimensions of SDG targets can be accelerated.

Table 2. Sustainable development goal (SDG) acceleration matrix.

\begin{tabular}{lccccc}
\hline \multirow{2}{*}{ Acceleration Area } & $\begin{array}{c}\text { Multiplier Effect } \\
\text { (\# of SDG Targets) }\end{array}$ & \multicolumn{4}{c}{ Balance across the 3 Dimensions } \\
\cline { 3 - 6 } Example: Female empowerment & 15 & Social & Economic & Environmental & Score \\
\hline Example: Impact investing & 10 & Yes & Yes & Yes & $15 \mid 3$ \\
\hline Example: Health records & 8 & Yes & Yes & No & $10 \mid 3$ \\
\hline
\end{tabular}

Source: Adapted from the SDG Accelerator and Bottleneck Assessment Tool [45]; the acceleration areas, multipliers, balances, and scores are examples and will depend on the specific context applicable to a given development intervention.

Our attempt here is to provide a simplified scorecard that will help development practitioners and organizations running blockchain experiments understand the bigger picture, pilot their interventions in a way to accelerate the achievement of the SDGs, and fulfill the sustainable development aspirations of individuals, communities, cities, regions, and governments worldwide. Inspired by the use of scorecards in the development practice, one of the experimental tools we have proposed in this work is the SDG Acceleration Scorecard (SAS). We argue that scorecards can be extremely useful in helping development practitioners monitor progress and measure results. To monitor capacity development, scorecards can be used as tools to quantify qualitative processes and measure the change in capacities using a set of relevant indicators to which specific ratings are assigned.

This was, for example, the case with the Capacity Development Scorecard developed to monitor the progress made towards achieving global environmental benefits for projects funded by the Global Environment Facility [46]. This scorecard was adapted to measure increases in capacity in the case of the implementation of the Access and Benefit Sharing (ABS) mechanism of the Nagoya Protocol, which governs the utilization of genetic resources and their associated traditional knowledge [47]. Simplified scorecard systems were also used to gather general information and conduct assessments. The WWF-World Bank Marine Protected Area (MPA) Scorecard, for instance, was designed for protected marine areas. It mainly relies on the available literature, in addition to the opinions of site managers and independent assessors [48]. Such a scorecard can be rapidly deployed, is less costly to implement, and broadly covers the relevant issues. Nevertheless, the depth of analysis provided by such tools remains low [49].

In our attempt to develop a scorecard to assess the SDG acceleration potential of blockchain-enabled solutions, we applied the core criteria defined in the thematic clusters to propose acceleration areas and tried to identify possible drivers of acceleration by asking specific questions to generate useful information that can be used for two main objectives. Under the first scenario, the scorecard can be used to assess the multiplier effect of a given blockchain-enabled solution in terms of how many SDGs are expected to benefit from such an intervention. The information generated by the scorecard can also be used to run a 
quick assessment to check whether or not the gaps in design and implementation have been properly addressed. In the second scenario, gathering data using the scorecard can enable a comparison between different blockchain-backed candidate solutions, in terms of their rational; risk category; the existence of proper mechanisms to generate data, track progress, assess outcomes, and manage change; and their overall ability to deliver tangible and holistic development outcomes.

The proposed SDG Acceleration Scorecard consists of acceleration areas, drivers, and a scoring system. To characterize the acceleration effect of a given blockchain-enabled solution, the scorecard provides descriptive sentences for each driver in the acceleration areas, each corresponding to a numerical rating from zero (0) to three (3) based on a 33-point scoring system. In the following paragraphs, we provide practical explanations related to the acceleration areas, the drivers within each area, and explain how the scores are assigned using descriptive sentences to describe each driver.

\section{A. Acceleration area 1: Use Case Development}

When use cases for blockchain-enabled solutions are properly designed by taking into consideration the interlinked nature of the SDGs, they can have a multiplier effect on the development dividends expected down the road. This in turn will boost the ongoing efforts to achieve the SDGs; hence, the acceleration effect.

\section{Driver A1}

This driver generates useful information to explain whether or not there is a strong justification for the design choice of a blockchain-backed solution. We propose asking the following or other alternative questions: Why would you consider blockchain as a solution to the problem encountered in your case?

Scorecard rating: We assigned scores of 0 to 3 to the responses provided:

0 -No response or not sure;

1-Blockchain is an emerging innovation in international development;

2-Blockchain has the potential to solve certain development challenges we face today;

3-Blockchain is the best alternative to address the specific challenges we are facing.

\section{Driver A2}

This driver generates useful information to explain whether or not a given blockchainbacked solution was compared to other alternatives. We propose asking the following or other alternative questions: Did you compare blockchain to other alternative solutions in terms of efficiency, scalability, performance, impact, and cost-effectiveness?

Scorecard rating: We assigned scores of 0 to 1 to the responses provided:

$0-\mathrm{No} /$ not sure;

$1-$ Yes.

\section{Driver A3}

This driver generates useful information to understand whether or not end-users were involved in testing and iterating a given blockchain-backed solution during the design, which is crucial in order to strengthen ownership and for consensus-building. We propose asking the following or other alternative questions: How many end-users were or will be approached during the design of your blockchain experiment?

Scorecard rating: We assigned scores of 0 to 3 to the responses provided:

0 -None or not sure;

1-Less than 2;

$2-2$ to 5 ;

3-More than 5.

Driver A4

This driver generates useful information to assess the multiplier effect of a given blockchain-powered solution in terms of its ability to generate wider development co- 
benefits by contributing to multiple SDGs. We propose asking the following or other alternative questions: How many SDGs do you expect your blockchain project to contribute to? Scorecard rating: We assigned scores of 0 to 3 to the responses provided:

0 -None or not sure;

1-Only 1 SDG;

2-From 2 to 3 SDGs;

3-Multiplier effect across more than 3 SDGs.

\section{B. Acceleration area 2: Risks and Ethical Considerations}

This part of the scorecard can generate risk-related information to reveal whether risks and ethical considerations related to blockchain technology are well understood and to ensure that a given blockchain experiment does not create more problems than the ones it was designed to solve.

\section{Driver B1}

This driver generates useful information to assess whether or not a risk analysis was undertaken for a given blockchain-powered solution. We propose asking the following or other alternative questions: Have you conducted a risk analysis to identify and assess the core risks associated with your blockchain experiment?

Scorecard rating: We assigned scores of 0 to 1 to the responses provided:

0 -No or not sure;

$1-$ Yes.

\section{Driver B2}

This driver generates useful information to assess the ability to identify and categorize risks in a structured way to enable a proper risk management response. As a follow-up to the question under B1, we propose asking the following or other alternative questions: What were the risks you have identified?

Scorecard rating: We assigned scores of 0 to 3 to the responses provided:

0 - None or not sure

1-Mostly external risks (high risk);

2-Mostly strategic risks (moderate risk);

3-Mostly preventable risks (low risk).

\section{Driver B3}

This driver generates useful information about whether or not there is a risk management mechanism for a given blockchain-powered solution to mitigate different types of risks. We propose asking the following or other alternative questions: Did you come up with a proper management response to mitigate the risks identified?

Scorecard rating: We assigned scores of 0 to 1 to the responses provided:

$0-$ No or not sure;

1 -Yes.

\section{Driver B4}

This driver generates useful information on whether or not there is a mechanism in place to update risk logs and detect emerging risks as blockchain-backed solutions are prototyped and deployed in a rapidly evolving legal, institutional, and technological landscape. We propose asking the following or other alternative questions: Did you set up a mechanism to detect and anticipate new emerging risks and address ethical considerations?

Scorecard rating: We assigned scores of 0 to 1 to the responses provided:

0 -No or not sure;

$1-$ Yes.

C. Acceleration area 3: Monitoring and Evaluation

This part of the scorecard can generate M\&E-related information to explain how activities will be monitored, how progress will be tracked, and which criteria will be used to evaluate the applicability and the outcomes of blockchain experiments. 


\section{Driver C1}

This driver generates useful information to assess the extent to which data are being collected for a given blockchain-powered solution. We propose asking the following or other alternative questions: Do you collect any type of data on your blockchain experiment?

Scorecard rating: We assigned scores of 0 to 3 to the responses provided:

0 -There is essentially no data collection;

1 -There is some sort of data collection;

2-Data are collected and analyzed;

3-Data are systematically collected, analyzed, and publicly shared to inform peers and decision-making.

\section{Driver C2}

This driver generates useful information to assess whether or not an M\&E mechanism is in place for a given blockchain-powered solution. We propose asking the following or other alternative questions: Do you have an $M \& E$ mechanism in place to ensure proper monitoring of your blockchain experiment?

Scorecard rating: We assigned scores of 0 to 3 to the responses provided:

0 -There is essentially no mechanism;

1 -There is some sort of mechanism;

2-There is a relatively good mechanism;

3-There is a well-functioning mechanism.

\section{Driver C3}

This driver generates useful information to explain the set of criteria used in assessing the applicability of blockchain experiments and evaluate their outcomes. We propose asking the following or other alternative questions: Which of the following criteria would you consider in evaluating your blockchain project? Performance, scalability, usability, security, cost, flexibility, transparency, legal regulations, environmental footprint, social impact, and SDG acceleration:

Scorecard rating: We assigned scores of 0 to 3 to the responses provided:

0 -None or not sure;

1-At least 1 criterion;

2-At least 3 criteria;

3-At least 5 criteria including the one on SDG acceleration.

D. Acceleration area 4: Innovation Management

The last part of the scorecard can be used to generate information to better understand the change dynamics in a given development setup and trigger adequate change management responses towards nurturing a culture of innovation and addressing pockets of resistance.

\section{Driver D1}

This driver generates useful information to assess whether or not there is some sort of awareness about change management. We propose asking the following or other alternative questions: Would you conduct any awareness-raising activities within your organization or team to visualize the potential of blockchain-enabled applications and co-create promising use cases?

Scorecard rating: We assigned scores of 0 to 1 to the responses provided:

0 -No or not sure;

1 -Yes.

\section{Driver D2}

This driver generates useful information to assess the willingness of managers to understand the root causes of resistance to change, and more specifically to blockchaindriven innovations. We propose asking the following or other alternative questions: Are you planning to explore the reasons behind any resistance that blockchain-backed solutions may face within your organization or team? 
Scorecard rating: We assigned scores of 0 to 3 to the responses provided:

0 -No or not sure;

$1-$ Yes.

Driver D3

This driver generates useful information to assess whether or not there are proper mechanisms in place to enable an innovation-centered environment. We propose asking the following or other alternative questions: Would you consider motivating your staff, partners, and end-users involved in your blockchain experiment?

Scorecard rating: We assigned scores of 0 to 3 to the responses provided:

0 -No or not sure;

1-Yes, by allocating appropriate time and resources for the team members to innovate and prototype promising solutions;

2-Yes, by investing in core capabilities and skills to implement blockchain projects through learning and practice;

3-Yes, through coaching for readiness to become a change agent.

\section{Driver D4}

This driver generates useful information to assess whether or not there is some sort of policy or strategy to maintain an innovation-prone set up in a given context. We propose asking the following or other alternative questions: Do you have a strategy to maintain an innovation-friendly environment within your organization?

Scorecard rating: We assigned scores of 0 to 3 to the responses provided:

0 -No or not sure;

1-Yes, by recommending corrective actions for improvement;

2-Yes, by measuring achievements;

3-Yes, by celebrating success.

Table 3 presents the SDG Acceleration Scorecard. We emphasize that the scoring system proposed here is not exclusive and that the scores assigned to each descriptive sentence can be revised and weighted accordingly on a case-by-case basis to better reflect the context within which a blockchain-backed solution is deployed. The scorecard can be used as a benchmark at the beginning of the design phase, but also to generate useful insights as the implementation of blockchain experiments is moving forward and to stay on track towards reaching the established targets. The SDG Acceleration Scorecard is a flexible tool and can be adapted and further improved to reflect the differentiated contexts underpinning blockchain experiments. 
Table 3. SDG Acceleration Scorecard.

Why would you consider blockchain as a solution to the problem encountered in your case?

Did you compare blockchain to other alternative solutions in terms of

efficiency, scalability, performance, impact, and cost-effectiveness?

How many end-users were/will be approached during the design of your

How many SDGs do you expect your blockchain project to contribute to?

Have you conducted a risk analysis to identify and assess the core risks associated with your blockchain experiment?

If yes, what were the risks you have identified?

Risks \& Ethical Considerations blockchain experiment?

0-No Response/Not sure

1 -Blockchain is an emerging innovation in

international development

2-Blockchain has the potential to solve some development

challenges we face today

3-Blockchain is the best alternative to address the specific challenges we are facing

\section{$0-$ No/Not sure}

1 -Yes

0 - None/Not sure

1 -Less than 2

$2-2$ to 5

3-More than 5

\section{0 - None/Not sure}

1 -Only 1 SDG

2-From 2 to 3 SDGs

3-Multiplier effect across more than 3 SDGs

\section{0 - No/Not sure}

1 -Yes

0-None/Not sure

1-Mostly external risks (High risk)

2-Mostly strategic risks (Moderate risk)

3-Mostly preventable risks (Low risk)

Did you come up with a proper management response to mitigate the

risks identified?

$0-\mathrm{No} /$ Not sure

$1-$ Yes

Did you set up a mechanism in place to detect and anticipate new

emerging risks and address ethical considerations?
$\mathrm{No} /$ Not sure

1 -Yes 
Table 3. Cont.

Acceleration Areas Drivers

\section{Scorecard}

0 - There is essentially no data collection

1 -There is some sort of data collection

Monitoring \& Evaluation

Innovation Management
2-Data are collected and analyzed

3-Data are systematically collected, analyzed, and publicly shared to inform peers and decision-making

Do you collect any type of data on your blockchain experiment?

Do you have an M\&E mechanism in place to ensure proper monitoring of your blockchain experiment?

Which of the following criteria would you consider in evaluating your

blockchain project? Performance, scalability, usability, security, cost,

flexibility, transparency, legal regulations, environmental footprint, socia impact, and SDG acceleration.

Would you conduct any awareness-raising activities within your organization or team to visualize the potential of blockchain-enabled applications and co-create promising ones?

Are you planning to explore the reasons behind any resistance that

blockchain-backed solutions may face within your organization or team?

0 - There is essentially no mechanism

1-There is some sort of mechanism

2-There is a relatively good mechanism

3-There is a well-functioning mechanism

0 -None/Not sure

1-At least 1 criterion

2-At least 3 criteria

3 -At least 5 criteria including the one on SDG acceleration

$0-$ No/Not sure

$1-$ Yes

\section{$0-\mathrm{No} /$ Not sure}

$1-$ Yes

\section{0 - No/Not sure}

1-Yes, by allocating appropriate time and resources for the team members to innovate and prototype promising solutions

2-Yes, by investing in core capabilities and skills to implement

blockchain projects through learning and practice

3 -Yes, through coaching for readiness to become a change agent

Do you have a strategy to maintain an innovation-friendly environmen within your organization?
$0-\mathrm{No} /$ Not sure

1-Yes, by recommending corrective actions for improvement

2-Yes, by measuring achievements

3-Yes, by celebrating success

Source: Authors. 
We fully recognize the limitations of using an SDG Acceleration Scorecard in the context of blockchain experiments. While it was designed as a low-cost and rapidly deployable tool, it should not be seen as a replacement for academically proven methodologies. The concept of scoring can entail risks of distortion, which leave ample room to improve accuracy by weighting the scores [38]. In this case, we assume that the weights assigned to each question in the SDG Acceleration Scorecard reflect some sort of rationale, which might not be necessarily the case. We considered a simple scoring system while recognizing its limitations.

Again, while recognizing the limitations of the proposed acceleration scorecard as a minimum viable product, which should not replace academically tested methodologies, the scorecard can help individuals and organizations to generate useful insights to guide their efforts throughout the journey of co-creating blockchain-powered solutions in the field of international development, and more specifically to meet the 2030 deadline for achieving the SDGs.

\subsection{Integrated Approach}

Intentional design is the recipe for developing promising blockchain applications. Unlike with other technologies, it is not easy to fix flows, alter records, or change contracts because of the immutable and distributed nature of data. By intentional design, here we mean an integrated approach that goes beyond identifying the problem faced and implementing an intervention to achieve expected outcomes; that is, going the extra mile to understand risks, ethical considerations, and technology choices that will usually make the difference.

As can be observed from Table 4, the prototype for the integrated approach proposed at this stage is a living tool that can be further improved as blockchain technology matures over the years to come, based on feedback from end-users as they domesticate and internalize the tool in their quest to adopt the technology and run experiments on blockchain-backed solutions in the international development space. 
Table 4. Integrated approach to using blockchain-enabled solutions as SDG accelerators.

\section{Factor-In Risks and Ethical Considerations}

Risks

We propose a three-step approach for risk

We propose the following stages to the blockchain use case development method:

- Understand blockchain

- $\quad$ Define the problem to be solved with blockchain

- Pitch your ideas

- $\quad$ Build consensus

- $\quad$ Prototype the solution

- Test and iterate
- $\quad$ Analyze (identify and assess core risks)

- Manage (develop proper responses)

- Analyze (detect new risks)

Ethical considerations

There are several ethical considerations with regards

to blockchain applications that need to be properly

addressed, such as environmental impacts, job

disruptions, or their potential illicit utilization

(criminality, weapons, drugs, money laundering, etc.)
3. Setup M\&E Frameworks

4. Enable Evolutionary Adaptation

Monitoring

We propose a basic three-strep approach for monitoring

blockchain projects:

- Collect data

- $\quad$ Analyze data

Evaluation

We provide sets of evaluation criteria to set up robust

$\begin{array}{ll}\text { We provide sets of evaluation criteria to set up robust } & \text { organizational level: } \\ \text { M\&E frameworks for blockchain projects and support a } & \text { - Unfreeze }\end{array}$

critical analysis while conducting evaluations: $\quad \bullet \quad$ Unfreeze

- Technical criteria
-
$\quad$ Refreeze the change

Change management

Organizations embracing blockchain

need to be change-adept. In the following

change management approach, the focus

is on change at the individual level as the

Source: Authors. 


\section{Discussion and Conclusions}

Can blockchain be a game-changer as an SDG accelerator? Or is it hype that is driven by high expectations for its applications? We argue that the power of blockchain can be effectively harnessed to deliver significant progress towards achieving SDGs. Nevertheless, we also argue that there are limitations to what can be done, hence the need to stress that development professionals cannot solve all issues around SDGs with blockchain and that implementation choices in designing and implementing blockchain-enabled solutions should be rigorously justified to demonstrate tangible added value compared to other alternative solutions, which might be less costly and less technical.

While we recognize the limitations in developing the SDG Acceleration Scorecard and the integrated approach to using blockchain-enabled solutions as SDG accelerators, these are meant to be minimally viable products and serve as living tools, making room for further improvements and finetuning as the technology matures and lessons learned from various experiments are widely shared across the international development space.

"Don't over-hype" and "don't over-promise" indeed remain key messages for individuals and organizations experimenting with blockchain-powered solutions in international development. We believe this work offers good food for thought to further stimulate the discussions within the research community, but also among innovation and development practitioners working at the frontlines to raise the double challenge of adapting to the new normal post-COVID19 and meeting the SDG targets by 2030 .

Author Contributions: Conceptualization, A.F.A., F.B. and M.D.; methodology, F.B.; software, F.B. and M.D.; validation, A.F.A., F.B. and M.D.; formal analysis, F.B.; investigation, A.F.A., F.B. and M.D.; resources, F.B. and M.D.; data curation, F.B. and M.D.; writing-original draft preparation, F.B.; writing-review and editing, A.F.A. and M.D.; visualization, F.B. and M.D.; supervision, A.F.A.; project administration, A.F.A. and M.D. All authors have read and agreed to the published version of the manuscript.

Funding: This research received no external funding.

Institutional Review Board Statement: Not applicable.

Informed Consent Statement: Not applicable.

Data Availability Statement: Not applicable.

Conflicts of Interest: The authors declare no conflict of interest.

\section{References}

1. Barbier, E.B.; Burgess, J.C. Sustainability and development after COVID-19. World Dev. 2020, 135, 105082. [CrossRef] [PubMed]

2. Upadhyay, N. Demystifying blockchain: A critical analysis of challenges, applications and opportunities. Int. J. Inf. Manag. 2020, 54, 102120. [CrossRef]

3. Zhu, S.; Song, M.; Lim, M.K.; Wang, J.; Zhao, J. The development of energy blockchain and its implications for China's energy sector. Resour. Policy 2020, 66, 101595. [CrossRef]

4. Jiang, L. The Age of Trust-The Problem Blockchain Solves That Others Cannot. 13 December 2018. Available online: https:/ / medium.com/swlh/the-age-of-trust-the-problem-blockchain-solves-that-others-cannot-6024ebf47cad (accessed on 24 September 2019).

5. Gartner. Digital Disruption Profile: Blockchain's Radical Promise Spans Business and Society. 2018. Available online: https: / / www.gartner.com/en/doc/3855708-digital-disruption-profile-blockchains-radical-promise-spans-business-and-society (accessed on 29 February 2020).

6. Mougayar, W. If You Understand Google Docs, You Can Understand Blockchain. Coindesk. 2016. Available online: https: / / www.coindesk.com/understand-google-docs-can-understand-blockchain (accessed on 18 November 2019).

7. Deloitte. Blockchain: A True Disruptor for the Energy Industry, Use Cases and Strategic Questions; Deloitte: New York, NY, USA, 2018.

8. Dalla Palma, S.; Pareschi, R.; Zappone, F. What is your Distributed (Hyper) Ledger? In Proceedings of the 4th International Workshop on Emerging Trends in Software Engineering for Blockchain (WETSEB'21) at ICSE 2021, Madrid, Spain, $23-29$ May 2021.

9. SelfKey. Understanding Public vs. Private Blockchain. HYPERLINK. 2020. Available online: https://selfkey.org/understandingpublic-vs-private-blockchain/ (accessed on 4 December 2019).

10. Jayachandran, P. The Difference between Public and Private Blockchain. 2017. Available online: IBM:Https://www.ibm.com/ blogs/blockchain/2017/05/the-difference-between-public-and-private-blockchain/ (accessed on 20 November 2019). 
11. Garriga, M.; Dalla Palma, S.; Arias, M.; de Renzis, A.; Pareschi, R.; Andrew Tamburri, D. Blockchain and cryptocurrencies: A classification and comparison of architecture drivers. Concurr. Comput. Pract. Exp. 2020, e5992. [CrossRef]

12. Massessi, D. Public vs. Private Blockchain in a Nutshell. 2018. Available online: Medium:Https://medium.com/coinmonks/ public-vs-private-blockchain-in-a-nutshell-c9fe284fa39f (accessed on 15 March 2021).

13. Uzsoki, D.; Guerdat, G. Impact Tokens: A Blockchain-Based Solution for Impact Investing. International Institute for Sustainable Development. 2019. Available online: https:/ /www.iisd.org/publications/impact-tokens-blockchain-based-solution-impactinvesting (accessed on 12 March 2021).

14. Sincock, C.; Lewis, R. ESG in Financial Services: Today and in the Future. Capco Intelligence. 2021. Available online: https: //www.capco.com/Intelligence/Capco-Intelligence/ESG-In-Financial-Services (accessed on 5 January 2020).

15. Fishcoin. A Blockchain Based Data Ecosystem for the Global Seafood Industry. 2018. Available online: https://fishcoin.co/files/ fishcoin.pdf (accessed on 24 November 2019).

16. DeCleene, J. How Blockchain-as-a-Service (Baas) Platforms are Speeding up Blockchain Adoption. Data Driven Investor. Available online: https:/ /www.datadriveninvestor.com/2018/02/17/how-blockchain-as-a-service-baas-platforms-are-speedingup-blockchain-adoption/\# (accessed on 30 May 2018).

17. Sin Kuang, L.; Xiwei, X.; Yin Kia, C.; Qinghua, L. Evaluating Suitability of Applying Blockchain. In Proceedings of the 2017 International Conference on Engineering of Complex Computer Systems, Fukuoka, Japan, 6-8 November 2017. [CrossRef]

18. Mckinsey. Blockchain beyond the Hype: What Is the Strategic Business Value? June 2018. Available online: https: //www.mckinsey.com/business-functions / digital-mckinsey/our-insights/blockchain-beyond-the-hype-what-is-thestrategic-business-value\# (accessed on 24 September 2019).

19. Fridgen, G.; Lockl, J.; Radszuwill, S.; Schweizer, A.; Urbach, N. A Solution in Search of a Problem: A Method for the Development of Blockchain Use. In Proceedings of the 24th Americas Conference on Information Systems (AMCIS), New Orleans, LA, USA, 16-18 August 2018.

20. Dam, R.; Siang, T. 5 Stages in the Design Thinking Process. The Interaction Design Foundation. 2019. Available online: https:/ / www.interaction-design.org/literature/article/5-stages-in-the-design-thinking-process (accessed on 15 October 2019).

21. Wachal, M. Factors to Evaluate When Using Blockchain Technology. 2019. Available online: http://scrypt.media/2019/08/08 / factors-to-evaluate-when-using-blockchain-technology/ (accessed on 17 February 2020).

22. Panetta, K. Blockchain Combines Innovation with Risk. Gartner, 16 October 2016. Available online: https://www.gartner.com/ smarterwithgartner/blockchain-combines-innovation-with-risk/(accessed on 5 December 2019).

23. Kaplan, S.R.; Mikes, A. Managing Risks: A New Framework. Harvard Business Review. June 2012. Available online: https: / / hbr.org/2012/06/managing-risks-a-new-framework (accessed on 14 November 2019).

24. Tasca, P. Managing Risk under the Blockchain Paradigm. Linkedin. Available online: https://www.linkedin.com/pulse/ managing-risk-under-blockchain-paradigm-paolo-tasca (accessed on 3 March 2017).

25. Thivaios, P. Managing the Risks of Blockchain. 2018. Available online: https:// knect365.com/riskminds/article/c515ed63-da124d4e-874a-eb83eefb3e66/managing-the-risks-of-blockchain (accessed on 1 October 2019).

26. CBECI. Cambridge Bitcoin Electricity Consumption Index. 2019. Available online: https://www.cbeci.org/ (accessed on 3 October 2019).

27. Houlding, D. Blockchain: 6 Key Ethical Considerations. Available online: Lifeboat:Https://lifeboat.com/blog/2019/01/ blockchain-6-key-ethical-considerations (accessed on 18 January 2019).

28. Longstaff, S. Blockchain: Some Ethical Considerations. The Ethics Center. Available online: https://ethics.org.au/blockchainsome-considerations/ (accessed on 16 March 2019).

29. Smith, J. Beyond Crypto-Blockchain Ethics. Hackernoon. Available online: https://hackernoon.com/beyond-cryptoblockchain-ethics-eabd8df6faf5 (accessed on 4 February 2019).

30. Apointe, C.; Fishbane, L. The Blockchain Ethical Design Framework. Beeck Center for Social Impact + Innovation-Georgetown University. 2018. Available online: https:/ / www.mitpressjournals.org/doi/pdf/10.1162/inov_a_00275 (accessed on 14 December 2019).

31. EPRS. Blockchain and the General Data Protection Regulation Can Distributed Ledgers Be Squared with European Data Protection Law? European Parliamentary Research Service. 2019. Available online: https://www.europarl.europa.eu/RegData/etudes/ STUD/2019/634445/EPRS_STU(2019)634445_EN.pdf (accessed on 17 March 2021).

32. Schwerin, S. Blockchain and privacy protection in the case of the european general data protection regulation (GDPR): A delphi study. J. Br. Blockchain Assoc. 2018, 1, 3554. [CrossRef]

33. OECD. DAC Principles for Evaluation of Development Assistance. Organisation for Economic Co-Operation and Development. 1991. Available online: https:/ / www.oecd.org/dac/evaluation/2755284.pdf (accessed on 16 October 2019).

34. Pasanen, T. 2018: Time to Update the DAC Evaluation Criteria? 2018. Available online: https://www.odi.org/blogs/10594-2018 -time-update-dac-evaluation-criteria (accessed on 4 October 2019).

35. Jiang, L. Breaking Blockchain-A Framework to Evaluate Blockchain Use Cases. 2018. Available online: https://hackernoon. com/breaking-blockchain-a-framework-to-evaluate-blockchain-use-cases-9efbc30a3fa7 (accessed on 5 December 2019).

36. WEF. Building Value with Blockchain Technology: How to Evaluate Blockchain's Benefits; World Economic Forum: Cologny, Switzerland, 2019.

37. Vangala, N. Evaluation Criteria for Blockchain. Medium, 27 June 2018. Available online: https://medium.com/nhct-nanohealthcare-token/evaluation-criteria-for-blockchain-e33a54b477aa(accessed on 23 September 2019). 
38. Fridgen, G.; Guggenmos, F.; Lockl, J.; Rieger, A.; Schweizer, A.; Urbach, N. Developing an Evaluation Framework for Blockchain in the Public Sector: The Example of the German Asylum Process. Amsterdam: Proceedings of the 1st ERCIM Blockchain Workshop 2018, Reports of the European Society for Socially Embedded Technologies (ISSN 2510-2591). 2018. Available online: https:/ / www.fim-rc.de/Paperbibliothek/Veroeffentlicht/756/wi-756.pdf (accessed on 30 May 2020).

39. Catalini, C.; Gans, J.S. Some Simple Economics of the Blockchain (No. w22952); National Bureau of Economic Research: Cambridge, MA, USA, 2016.

40. Swan, M. Blockchain: Blueprint for a New Economy; O'Reilly Media, Inc.: Newton, MA, USA, 2015.

41. Narayan, R.; Tidström, A. Tokenizing coopetition in a blockchain for a transition to circular economy. J. Clean. Prod. 2020, 263, 121437. [CrossRef]

42. Abrahamson, E.; Rosenkopf, L. Social network effects on the extent of innovation diffusion: A computer simulation. Organ. Sci. 1997, 8, 289-309. [CrossRef]

43. Prosci. What Is the ADKAR Model? 2019. Available online: https://www.prosci.com/adkar/adkar-model (accessed on 3 October 2019).

44. Talib Hussain, S.; Shen, L.; Akram, T.; Jamal Haider, M.; Hadi Hussain, S.; Ali, M. Kurt Lewin's Change Model: A Critical Review of the Role of Leadership and Employee Involvement in Organizational Change. J. Innov. Knowl. 2016. Available online: https: / / study.com/academy/lesson/lewins-3-stage-model-of-change-unfreezing-changing-refreezing.html (accessed on 21 November 2019).

45. UNDP. SDG Accelerator and Bottleneck Assessment. 2017. Available online: https://www.undp.org/content/dam/undp/ library/SDGs/English/SDG_Accelerator_and_Bottleneck_Assessment_Tool.pdf (accessed on 29 March 2020).

46. Bellamy, J.-J.; Hill, K. Monitoring Guidelines of Capacity Development in Global Environment Facility Projects. Global Support Programme, Bureau for Development Policy, United Nations Development Programme. 2010. Available online: https://www. thegef.org/sites/default/files/publications/Monitoring_Guidelines_Report-final.pdf (accessed on 7 January 2020).

47. CBD. Overview of Access and Benefit-Sharing Capacity-Building Tools and Resources. Convention on Biological Diversity. 2018. Available online: https:/ /www.cbd.int/doc/c/99fa/59d2/f895959bebd76275b1fbf283/abs-cbiac-2018-01-02-add2-en.pdf (accessed on 8 February 2020).

48. Leverington, F.; Hockings, M.; Lemos, K.; Courrau, J.; Pavese, H. Management Effectiveness Evaluation in Protected Areas, a Global Study, Overview of Approaches and Methodologies; Supplementary Report No.1, The University of Queensland: Gatton, Australia, 2008.

49. Staub, F.; Hatziolos, M. Score Card to Assess Progress in Achieving Management Effectiveness Goals for Marine Protected Areas, Revised Version; The World Bank: Washington, DC, USA, 2004. 